\title{
An IDH1-mutated primary gliosarcoma: case report
}

\author{
Jason K. Hsieh, BS, ${ }^{1,2}$ Christopher S. Hong, BA, ${ }^{3}$ Sunil Manjila, MD, Mark L. Cohen, MD, ${ }^{5}$ \\ Simon Lo, MD, ${ }^{1,6,7}$ Lisa Rogers, DO, ${ }^{1,6,8}$ and Andrew E. Sloan, MD ${ }^{1,4,6}$
}

\begin{abstract}
${ }^{1}$ Case Western Reserve University School of Medicine, Cleveland; ${ }^{2}$ Cleveland Clinic Lerner College of Medicine, Cleveland; ${ }^{3}$ The Ohio State University School of Medicine, Columbus; ${ }^{4}$ Department of Neurological Surgery, University Hospitals-Case Medical Center, Cleveland; ${ }^{5}$ Department of Pathology, and ${ }^{6}$ Case Comprehensive Cancer Center, Case Western Reserve University School of Medicine, Cleveland; and 7 Departments of Radiation Oncology and ${ }^{8}$ Neurology, University Hospitals-Seidman Cancer Center, Cleveland, Ohio
\end{abstract}

\begin{abstract}
The authors present the case of a primary gliosarcoma with an isocitrate dehydrogenase-1 (IDH1) mutation. A 75-yearold man presented with a 3-day history of multiple focal seizures and was found on MRI to have a 2.2-cm left parietal enhancing mass lesion. Brain MRI for tremor performed 8 years prior to this presentation was normal. En bloc resection revealed a high-grade glioma with sarcomatous components that was immunoreactive for the $\mathrm{R} 132 \mathrm{H}$ variant of IDH1 by antibody. Gliosarcoma is a rare variant of glioblastoma that arises most frequently as a primary tumor, and has equal or worse survival and an increased propensity for extracranial metastases compared with other Grade 4 gliomas. In contrast, isocitrate dehydrogenase- 1 and -2 mutations are associated with low-grade gliomas with increased survival and less commonly with glioblastoma. To the authors' knowledge, there has been only 1 other published report of a primary gliosarcoma carrying an isocitrate dehydrogenase mutation. This rare genetic-histological combination highlights potential differences between glioblastoma and gliosarcoma and may warrant additional study.
\end{abstract}

http://thejns.org/doi/abs/10.3171/2016.2.JNS151482

KEY WORDS gliosarcoma; isocitrate dehydrogenase; glioblastoma; oncology

I SOCITRATE dehydrogenase-1 (IDHI) mutations were first identified in glioblastoma by Parsons and colleagues in $2008,^{30}$ and were discovered to be particularly prevalent in younger patients and secondary glioblastomas. Notably, patients with IDHI mutation-positive gliomas demonstrated strikingly increased overall survival compared with those with IDHI wild-type tumors, a finding corroborated in 2 seminal studies ${ }^{35,41}$ among many others. Subsequent work has demonstrated that IDHI mutations occur early in tumorigenesis, preceding further genetic alterations including TP53 loss and $1 \mathrm{p} / 19 \mathrm{q}$ codeletion..$^{18}$ IDHI mutations have since come to differentiate secondary glioblastoma from primary glioblastoma, the latter characterized by IDHI wild-type status and early phosphatase and tensin homolog loss and epidermal growth factor receptor (EGFR) amplification..$^{14}$ IDHI mutationpositive tumors diagnosed as primary glioblastoma have been proposed to be glioblastomas that progressed rapidly from a previously undiagnosed lower-grade glioma. ${ }^{27,29}$

Gliosarcoma is a relatively uncommon pathological variant of glioblastoma characterized by mixed glial and mesenchymal components. It comprises approximately $2.2 \%$ of glioblastomas, ${ }^{11,17,24}$ arising most often as a pri- mary tumor. ${ }^{21}$ Compared with glioblastoma, gliosarcoma is found more frequently in the temporal lobes ${ }^{16,26}$ and may have an increased predilection for local invasion and extracranial metastasis. , $^{3,10,22,23,25,28,31,32}$ It often presents surgically as a firm, superficial lesion adherent to the meninges that may be mistaken intraoperatively for a meningioma. ${ }^{3,26}$

Although most gliosarcomas are diagnosed at the time of surgery and are believed to arise de novo (primary gliosarcoma), ${ }^{6}$ a small fraction of gliosarcomas develop after cranial irradiation for glioblastoma or other cranial lesions, and an even smaller fraction develop from lowergrade precursors in the absence of radiation or other intervention. ${ }^{6,13,38}$ For instance, in 1 series by Perry and colleagues $^{30}$ of 32 gliosarcomas, 25 were considered primary tumors whereas the remaining 7 cases occurred after irradiation, and in a series of 30 secondary gliosarcomas by Han and colleagues, ${ }^{12}$ only 1 case developed from a primary glioblastoma after resection in the absence of adjuvant external beam radiation or Gamma Knife radiosurgery.

To date, there exists only 1 report of a putatively primary gliosarcoma with $I D H 1$ mutation positivity, ${ }^{19}$ whereas secondary gliosarcoma in the absence of radiation treatment is exceedingly rare. In this paper we present the case of an

ABBREVIATIONS EGFR = epidermal growth factor receptor; GFAP = glial fibrillary acidic protein; IDH1 = isocitrate dehydrogenase-1.

SUBMITTED June 24, 2015. ACCEPTED February 5, 2016.

INCLUDE WHEN CITING Published online May 6, 2016; DOI: 10.3171/2016.2.JNS151482. 
elderly gentleman with no history of previous intracranial mass lesions who presented to the hospital with new-onset seizures and was found to have an $I D H I$ mutation-positive gliosarcoma.

\section{Case Report}

History and Presentation

A 75-year-old man presented to an outside hospital with a 3-day history of multiple focal seizures manifesting as episodes of right hand and arm numbness, weakness, and pain. Of note, approximately 8 years before this presentation he had experienced a transient episode of right arm tremor and underwent brain MRI, which revealed no abnormal mass lesions. His past medical, surgical, family, and social histories were otherwise noncontributory. He underwent a CT scan that revealed a left parietal lesion, and was transferred to our institution (University Hospitals-Case Medical Center and the Seidman Cancer Center) under the care of the neurosurgical service.

On admission, the patient endorsed right arm pain and numbness as well as word finding difficulties. On physical examination he was grossly intact aside from mild hypoesthesia in the right upper extremity, mild global hyperreflexia, and altered extraocular pursuit movements likely secondary to medication. The patient underwent volumetric MRI that confirmed the presence of a rounded $2.2 \times 2.2-\mathrm{cm}$ left parietal mass with surrounding hazy hyperintensity on FLAIR and T2-weighted images, potentially representing either vasogenic edema or nonenhancing infiltrating tumor. Postcontrast images revealed irregular enhancement within the mass and probable necrosis. A CT PET scan demonstrated peripheral intensely hypermetabolic activity within the lesion with relatively decreased activity centrally, but otherwise found no evidence of extracranial lesions.

\section{Operation and Postoperative Course}

Left occipitoparietal craniotomy with stereotactic computer-aided navigation and electrophysiological motor mapping was performed. After electrophysiological determination that the motor cortex lay at least 1 gyrus anterior to the anterior margin of the tumor, a sulcal plane was opened under the microscope and a biopsy of the tumor was taken, which revealed a high-grade glioma. The sulci around the tumor were opened and the tumor was grossly removed in 1 piece. The patient recovered without complication and was discharged from the hospital on postoperative Day 2.

Postoperative MRI showed gross-total resection. At the 1-month follow-up, the patient had recovered well from surgery, with mild persistent sensory changes in his right arm and grossly normal speech with occasional word-finding difficulty. The final pathology revealed a high-grade astrocytic tumor; glial fibrillary acidic protein (GFAP) and reticulin stains confirmed sarcomatous differentiation within the tumor, and it was immunoreactive for IDH1R132H (Fig. 1).

The patient received adjuvant temozolomide therapy and 6000 cGy external beam radiation therapy to the surgical bed in 30 fractions. At his most recent follow-up evaluation approximately 7 months after surgery, the patient continued to do well with some mild sequelae in the form of steroid myopathy and an isolated seizure. Brain MRI at that time revealed increased irregular enhancement, without increased mass effect, around the resection cavity that could represent progression or pseudoprogression of his disease.

\section{Discussion}

This unusual case highlights our evolving understanding of the molecular genetics of glioblastoma and its rare variant, gliosarcoma. Although gliosarcoma resembles glioblastoma with regards to clinical presentation and is often managed under similar or identical protocols, gliosarcoma may be a unique clinicopathological entity.,39 For instance, gliosarcoma may have an increased propensity for local and systemic invasive metastasis, and while data suggest that patients derive benefit from resection and aggressive treatment under existing glioblastoma-directed temozolomide- and radiotherapy-based protocols, ${ }^{32,39}$ survival for primary gliosarcoma may be worse than for primary glioblastoma. 7,17,21

Sequencing and comparative genomic hybridization studies of microdissected gliosarcoma have revealed that tumor cells from its glial and mesenchymal components contain identical alterations in specific genes, suggesting that both tumor compartments arise from the same precursor. ${ }^{4,5}$ However, despite having a common clonal cell of origin, the 2 compartments may differ substantially in their genetic alterations. In 1 study by Actor and colleagues, only $57 \%$ of chromosomal imbalances were shared by both glial and mesenchymal subpopulations. ${ }^{1}$ Compared with primary glioblastoma, gliosarcomas in this study had a smaller number of chromosomes involved in imbalances, and though not significant, more gliosarcomas exhibited a loss on chromosomes $9 \mathrm{p}$ and fewer on chromosome 10 and 22 than glioblastoma. ${ }^{1}$ The majority of gliosarcomas had a gain on chromosome 7 (15/20 analyzed), possibly highlighting a role of chromosome 7 in the evolution of gliosarcoma.

Another study by Reis and colleagues of 19 gliosarcomas also found an increased frequency of gains on chromosome 7, as well as a complete absence of EGFR amplification. ${ }^{33}$ It is not known at what stage in tumorigenesis the glial and mesenchymal components of gliosarcoma diverge; however, the genetic and clinical differences between gliosarcoma and glioblastoma raise the possibility that gliosarcoma may warrant consideration as an independent clinical entity.

As mentioned earlier, IDH1 mutations play an important role in the evolution of gliomas. The majority (approximately $90 \%$ ) of $I D H 1$ mutations involve the substitution of histidine for arginine at position 132 (R132H). Rarely, IDH2 mutations occur instead, affecting the arginine residue at codon 172, representing the structural analog of the R132 position in IDH1.2,42 IDH1/2-mutated subunits form heterodimers with their wild-type counterparts to catalyze the conversion of the citric acid cycle metabolite, alpha-ketoglutarate, into D-2-hydroxyglutarate in an nicotinamide adenine dinucleotide diphosphate-depen- 


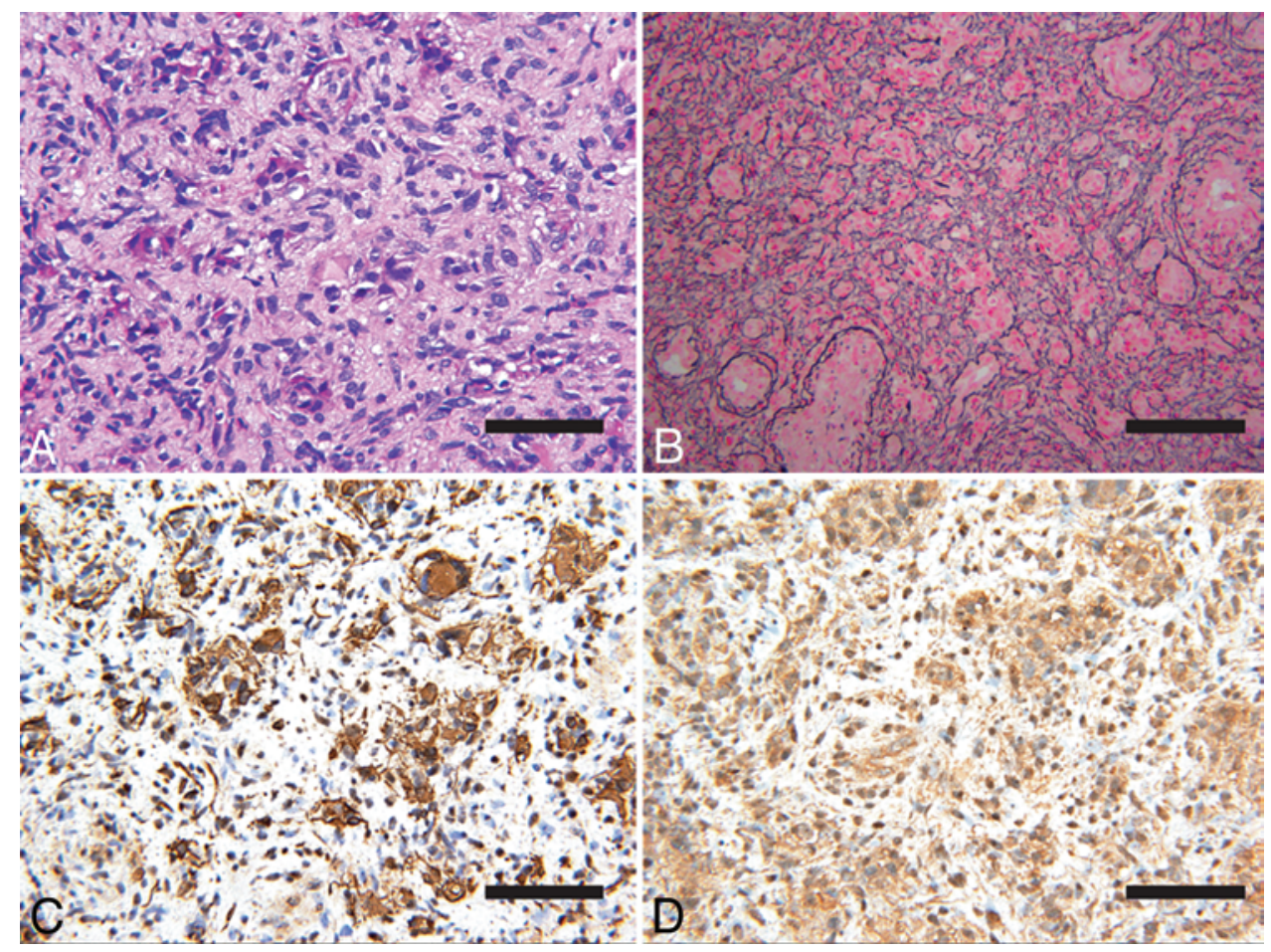

FIG. 1. Photomicrographs. A: H \& E stain showing nests of astrocytic cells within a dense collagenous stroma. B: Reticulin stain disclosing a dense pericellular reticulin network throughout the tumor. C: GFAP stain highlighting the astrocytic elements embedded within the collagenous matrix. D: Positive immunoreactivity for mutant IDH-1 R132H. Bar $=100 \mu \mathrm{m}$. Figure is available in color online only.

dent manner. ${ }^{8}$ Accumulation of D-2-hydroxglutarate leads to inhibition of the functionally diverse family of 2-oxoglutarate oxygenases, resulting in downstream oncogenic consequences, including maintenance of a hypermethylator phenotype, perturbation of collagen maturation and basement membrane function, induction of the HIF-1 $\alpha$ pathway, and increased oxidative damage. . $^{36,37,40,41,43}$

Whether IDH mutations play a role in the evolution of gliosarcoma is unknown. In a few case reports of secondary gliosarcoma arising from oligodendroglioma ("oligo- sarcoma"), IDH mutations were identified. ${ }^{15,38} I D H 1$ and TP53 mutation analysis were used in 1 case report of a secondary gliosarcoma to demonstrate that it likely arose from a previous anaplastic astrocytoma. ${ }^{33}$ To date, we have found only 1 report of a primary gliosarcoma with $I D H I$ positivity in a series of 25 gliosarcomas; interestingly, this tumor also contained a focal oligodendroglial component.$^{19}$ A review of studies investigating IDH mutations in gliosarcomas is presented in Table 1.

Secondary gliosarcoma is exceptionally rare, and among

TABLE 1. Previous reports investigating IDH status in gliosarcomas*

\begin{tabular}{llcll}
\hline \multicolumn{1}{c}{ Authors \& Year } & \multicolumn{1}{c}{ Study Design } & $\begin{array}{c}\text { No. of } \\
\text { Gliosarcomast }\end{array}$ & \multicolumn{1}{c}{ Detection Method } & IDH1 Mutant Gliosarcomas \\
\hline Balss et al., 2008 & $\begin{array}{c}\text { Retrospective 685-case } \\
\text { series }\end{array}$ & 5 & Sequencing of IDH1 (codon 132) & None detected \\
\hline Lusis et al., 2010 & Retrospective 3-case series & 2 & Immunohistochemistry & None detected \\
\hline Romeike et al., 2011 & Case report & 1 & $\begin{array}{c}\text { Immunohistochemistry \& sequencing } \\
\text { of IDH1 }\end{array}$ & 1 secondary tumor (after treatment) \\
\hline Vajtai et al., 2012 & Case report & 1 & Immunohistochemistry & 1 secondary tumor (after treatment) \\
\hline Lee et al., 2012 & Retrospective 26-case series & 26 & $\begin{array}{c}\text { Sequencing of IDH1 (codon 132) \& } \\
\text { IDH2 (codon 172) }\end{array}$ & $\begin{array}{c}\text { 1 primary tumor, 1 secondary tumor } \\
\text { (after treatment) }\end{array}$ \\
\hline Hiniker et al., 2013 & Case report & 1 & Immunohistochemistry & 1 secondary tumor (after treatment) \\
\hline Joseph et al., 2013 & Retrospective 167-case series & 45 & Immunohistochemistry & 3/23 secondary tumors; 0/22 primary \\
tumors
\end{tabular}

* Studies include those specifically investigating gliosarcoma and studies of other brain tumors that also reported the presence of gliosarcoma.

$\dagger$ Number assessed for IDH status in each study. 
secondary gliosarcomas, the vast majority arise from glioblastoma or other intracranial lesions in the context of irradiation. As such, $I D H 1$ mutation positivity in gliosarcomas may reflect the early time course of IDHI mutations in gliomagenesis. It is unknown whether primary gliosarcoma may harbor IDH mutations, in contrast to primary glioblastoma. Our patient presented to the hospital, in the context of previous negative head imaging, with a new mass lesion that was clinically diagnosed as a primary tumor. Whether his lesion was a rare primary gliosarcoma with IDH positivity, or a gliosarcoma that arose from a lower-grade precursor in the absence of irradiation, is unclear. Although gliosarcoma shares many features with glioblastoma, its unique genetic and clinical aspects may offer opportunities for alternative treatment and consideration beyond that offered for glioblastoma.

\section{References}

1. Actor B, Cobbers JMJL, Büschges R, Wolter M, Knobbe CB, Lichter P, et al: Comprehensive analysis of genomic alterations in gliosarcoma and its two tissue components. Genes Chromosomes Cancer 34:416-427, 2002

2. Balss J, Meyer J, Mueller W, Korshunov A, Hartmann C, von Deimling A: Analysis of the IDH1 codon 132 mutation in brain tumors. Acta Neuropathol 116:597-602, 2008

3. Beaumont TL, Kupsky WJ, Barger GR, Sloan AE: Gliosarcoma with multiple extracranial metastases: case report and review of the literature. J Neurooncol 83:39-46, 2007

4. Biernat W, Aguzzi A, Sure U, Grant JW, Kleihues P, Hegi ME: Identical mutations of the p53 tumor suppressor gene in the gliomatous and the sarcomatous components of gliosarcomas suggest a common origin from glial cells. J Neuropathol Exp Neurol 54:651-656, 1995

5. Boerman RH, Anderl K, Herath J, Borell T, Johnson N, Schaeffer-Klein J, et al: The glial and mesenchymal elements of gliosarcomas share similar genetic alterations. J Neuropathol Exp Neurol 55:973-981, 1996

6. Codispoti KET, Mosier S, Ramsey R, Lin MT, Rodriguez FJ: Genetic and pathologic evolution of early secondary gliosarcoma. Brain Tumor Pathol 31:40-46, 2014

7. Damodaran O, van Heerden J, Nowak AK, Bynevelt M, McDonald K, Marsh J, et al: Clinical management and survival outcomes of gliosarcomas in the era of multimodality therapy. J Clin Neurosci 21:478-481, 2014

8. Dang L, White DW, Gross S, Bennett BD, Bittinger MA, Driggers EM, et al: Cancer-associated IDH1 mutations produce 2-hydroxyglutarate. Nature 462:739-744, 2009

9. Dawar R, Fabiano AJ, Qiu J, Khushalani NI: Secondary gliosarcoma with extra-cranial metastases: a report and review of the literature. Clin Neurol Neurosurg 115:375-380, 2013

10. Demirci S, Akalin T, Islekel S, Ertan Y, Anacak Y: Multiple spinal metastases of cranial gliosarcoma: a case report and review of the literature. J Neurooncol 88:199-204, 2008

11. Galanis E, Buckner JC, Dinapoli RP, Scheithauer BW, Jenkins RB, Wang CH, et al: Clinical outcome of gliosarcoma compared with glioblastoma multiforme: North Central Cancer Treatment Group results. J Neurosurg 89:425-430, 1998

12. Han SJ, Yang I, Otero JJ, Ahn BJ, Tihan T, McDermott MW, et al: Secondary gliosarcoma after diagnosis of glioblastoma: clinical experience with 30 consecutive patients. J Neurosurg 112:990-996, 2010

13. Han SJ, Yang I, Tihan T, Chang SM, Parsa AT: Secondary gliosarcoma: a review of clinical features and pathological diagnosis. J Neurosurg 112:26-32, 2010

14. Hartmann C, Hentschel B, Simon M, Westphal M, Schackert $\mathrm{G}$, Tonn JC, et al: Long-term survival in primary glioblasto- ma with versus without isocitrate dehydrogenase mutations. Clin Cancer Res 19:5146-5157, 2013

15. Hiniker A, Hagenkord JM, Powers MP, Aghi MK, Prados MD, Perry A: Gliosarcoma arising from an oligodendroglioma (oligosarcoma). Clin Neuropathol 32:165-170, 2013

16. Joseph NM, Phillips J, Dahiya S, M Felicella M, Tihan T, Brat DJ, et al: Diagnostic implications of IDH1-R132H and OLIG2 expression patterns in rare and challenging glioblastoma variants. Mod Pathol 26:315-326, 2013

17. Kozak KR, Mahadevan A, Moody JS: Adult gliosarcoma: epidemiology, natural history, and factors associated with outcome. Neuro Oncol 11:183-191, 2009

18. Lai A, Kharbanda S, Pope WB, Tran A, Solis OE, Peale F, et al: Evidence for sequenced molecular evolution of IDH1 mutant glioblastoma from a distinct cell of origin. J Clin Oncol 29:4482-4490, 2011

19. Lee D, Kang SY, Suh YL, Jeong JY, Lee JI, Nam DH: Clinicopathologic and genomic features of gliosarcomas. J Neurooncol 107:643-650, 2012

20. Lusis EA, Travers S, Jost SC, Perry A: Glioblastomas with giant cell and sarcomatous features in patients with Turcot syndrome type 1: a clinicopathological study of 3 cases. Neurosurgery 67:811-817, 2010

21. Lutterbach J, Guttenberger R, Pagenstecher A: Gliosarcoma: a clinical study. Radiother Oncol 61:57-64, 2001

22. Maeda D, Miyazawa T, Toyooka T, Shima K: Temporal gliosarcoma with extraneural metastasis: case report. Neurol Med Chir (Tokyo) 50:343-345, 2010

23. Mansouri B, Barboriak DP, Kilani RK: Gliosarcoma metastatic to the leptomeninges and dura. J Neuroimaging 23:245-247, 2013

24. Meis JM, Martz KL, Nelson JS: Mixed glioblastoma multiforme and sarcoma. A clinicopathologic study of 26 radiation therapy oncology group cases. Cancer 67:2342-2349, 1991

25. Mesfin FB, Deshaies EM, Patel R, Weaver S, Spurgas P, Popp AJ: Metastatic gliosarcoma with a unique presentation and progression: case report and review of the literature. Clin Neuropathol 29:147-150, 2010

26. Morantz RA, Feigin I, Ransohoff J III: Clinical and pathological study of 24 cases of gliosarcoma. J Neurosurg 45:398-408, 1976

27. Nobusawa S, Watanabe T, Kleihues P, Ohgaki H: IDH1 mutations as molecular signature and predictive factor of secondary glioblastomas. Clin Cancer Res 15:6002-6007, 2009

28. Oberndorfer S, Wöhrer A, Hainfellner JA, Calabek B, Tinchon A, Brandl I, et al: Secondary gliosarcoma with massive invasion of meninges, skull base, and soft tissue, and systemic metastasis. Clin Neuropathol 32:522-524, 2013

29. Ohgaki H, Kleihues P: The definition of primary and secondary glioblastoma. Clin Cancer Res 19:764-772, 2013

30. Parsons DW, Jones S, Zhang X, Lin JCH, Leary RJ, Angenendt $\mathrm{P}$, et al: An integrated genomic analysis of human glioblastoma multiforme. Science 321:1807-1812, 2008

31. Perry JR, Ang LC, Bilbao JM, Muller PJ: Clinicopathologic features of primary and postirradiation cerebral gliosarcoma. Cancer 75:2910-2918, 1995

32. Rapp M, Felsberg J, Sorg RV, Gerharz CD, Sabel M: Case report: extracranial metastasis from gliosarcoma-the influence of immune system. Br J Neurosurg 25:286-288, 2011

33. Reis RM, Könü-Lebleblicioglu D, Lopes JM, Kleihues P, Ohgaki H: Genetic profile of gliosarcomas. Am J Pathol 156:425-432, 2000

34. Romeike BFM, Chen Y, Walter J, Petersen I: Diagnostic utility of IDH1- and p53-mutation analysis in secondary gliosarcoma. Clin Neuropathol 30:231-234, 2011

35. Sanson M, Marie Y, Paris S, Idbaih A, Laffaire J, Ducray F, et al: Isocitrate dehydrogenase 1 codon 132 mutation is an 
important prognostic biomarker in gliomas. J Clin Oncol 27:4150-4154, 2009

36. Sasaki M, Knobbe CB, Itsumi M, Elia AJ, Harris IS, Chio IIC, et al: D-2-hydroxyglutarate produced by mutant IDH1 perturbs collagen maturation and basement membrane function. Genes Dev 26:2038-2049, 2012

37. Turcan S, Rohle D, Goenka A, Walsh LA, Fang F, Yilmaz E, et al: IDH1 mutation is sufficient to establish the glioma hypermethylator phenotype. Nature 483:479-483, 2012

38. Vajtai I, Vassella E, Hewer E, Kappeler A, Reinert MM: Sarcomatous evolution of oligodendroglioma ("oligosarcoma"): confirmatory report of an uncommon pattern of malignant progression in oligodendroglial tumors. Pathol Res Pract 208:750-755, 2012

39. Walker GV, Gilbert MR, Prabhu SS, Brown PD, McAleer MF: Temozolomide use in adult patients with gliosarcoma: an evolving clinical practice. J Neurooncol 112:83-89, 2013

40. Watanabe T, Nobusawa S, Kleihues P, Ohgaki H: IDH1 mutations are early events in the development of astrocytomas and oligodendrogliomas. Am J Pathol 174:1149-1153, 2009

41. Xu W, Yang H, Liu Y, Yang Y, Wang P, Kim SH, et al: Oncometabolite 2-hydroxyglutarate is a competitive inhibitor of $\alpha$-ketoglutarate-dependent dioxygenases. Cancer Cell 19:17-30, 2011

42. Yan H, Parsons DW, Jin G, McLendon R, Rasheed BA, Yuan W, et al: IDH1 and IDH2 mutations in gliomas. N Engl J Med 360:765-773, 2009
43. Zhao S, Lin Y, Xu W, Jiang W, Zha Z, Wang P, et al: Gliomaderived mutations in IDH1 dominantly inhibit IDH1 catalytic activity and induce HIF-1alpha. Science 324:261-265, 2009

\section{Disclosures}

The authors report no conflict of interest concerning the materials or methods used in this study or the findings specified in this paper.

\section{Author Contributions}

Conception and design: Sloan, Hsieh, Manjila. Acquisition of data: Hsieh, Manjila, Cohen. Analysis and interpretation of data: Hsieh. Drafting the article: Sloan, Hsieh, Hong. Critically revising the article: all authors. Reviewed submitted version of manuscript: Hsieh, Hong, Manjila, Cohen, Lo, Rogers. Approved the final version of the manuscript on behalf of all authors: Sloan. Study supervision: Sloan.

\section{Correspondence}

Andrew Sloan, Department of Neurological Surgery, University Hospitals-Case Medical Center and Case Comprehensive Cancer Center, 11100 Euclid Ave., HAN 524, Cleveland, OH 44106. email: andrew.sloan@uhhospitals.org. 